\title{
Three-Dimensional Polyhedra Can Be Described by Three Polynomial Inequalities
}

\author{
Gennadiy Averkov • Martin Henk
}

Received: 14 July 2008 / Revised: 28 March 2009 / Accepted: 8 April 2009 /

Published online: 6 May 2009

(C) Springer Science+Business Media, LLC 2009

\begin{abstract}
Bosse et al. conjectured that for every natural number $d \geq 2$ and every $d$-dimensional polytope $P$ in $\mathbb{R}^{d}$, there exist $d$ polynomials $p_{1}(x), \ldots, p_{d}(x)$ satisfying $P=\left\{x \in \mathbb{R}^{d}: p_{1}(x) \geq 0, \ldots, p_{d}(x) \geq 0\right\}$. We show that every three-dimensional polyhedron can be described by three polynomial inequalities, which confirms the conjecture for the case $d=3$ but also provides an analogous statement for the case of unbounded polyhedra. The proof of our result is constructive.
\end{abstract}

Keywords Łojasiewicz's inequality · Polynomial · Polytope - Semi-algebraic set · Theorem of Bröcker and Scheiderer

\section{Introduction}

A subset $S$ of $\mathbb{R}^{d}(d \in \mathbb{N})$ is said to be an elementary closed semi-algebraic set in $\mathbb{R}^{d}$ if

$$
S=\left(q_{1}, \ldots, q_{m}\right)_{\geq 0}:=\left\{x \in \mathbb{R}^{d}: q_{1}(x) \geq 0, \ldots, q_{m}(x) \geq 0\right\}
$$

for some $m \in \mathbb{N}$ and polynomials $q_{1}(x), \ldots, q_{m}(x)$ over $\mathbb{R}$. Every elementary closed semi-algebraic set $S$ in $\mathbb{R}^{d}$ can be represented by $d(d+1) / 2$ polynomial inequalities, i.e., there exist polynomials $p_{1}(x), \ldots, p_{n}(x)$ with $n \leq d(d+1) / 2$ such that

$$
S=\left(p_{1}, \ldots, p_{n}\right)_{\geq 0} .
$$

Work supported by the German Research Foundation within the Research Unit 468 "Methods from Discrete Mathematics for the Synthesis and Control of Chemical Processes".

G. Averkov $\cdot$ M. Henk $(\bowtie)$

Institut für Algebra und Geometrie, Fakultät für Mathematik, Otto-von-Guericke-Universität Magdeburg, Universitätsplatz 2, 39106 Magdeburg, Germany

e-mail: martin.henk@mathematik.uni-magdeburg.de

G. Averkov

e-mail: gennadiy.averkov@ovgu.de 
This follows from the well-known result of Bröcker and Scheiderer; see [4, Sects. 6.5 and 10.4], [1, p. 143]. However, the arguments of Bröcker and Scheiderer are highly nonconstructive, so that not much is known about the relationship of the polynomials $q_{1}(x), \ldots, q_{m}(x)$ and $p_{1}(x), \ldots, p_{n}(x)$. One can consider the following more specific problem. Given a special class $\mathcal{S}$ of elementary closed semi-algebraic sets in $\mathbb{R}^{d}$, find the minimal $n$ such that every semi-algebraic set $S$ from $\mathcal{S}$ can be represented by $n$ polynomials $p_{1}(x), \ldots, p_{n}(x)$ and, moreover, find algorithms for determination of $p_{1}(x), \ldots, p_{n}(x)$ from $q_{1}(x), \ldots, q_{m}(x)$; see also $[3,9]$.

Results on representations of semi-algebraic sets are relevant for several research areas including polynomial and semi-definite optimization; see [10, 11]. In this manuscript we are concerned with polynomial representations of polyhedra. A nonempty subset $P$ of $\mathbb{R}^{d}$ is said to be a polyhedron if $P$ is the intersection of a finite number of closed halfspaces; see $[8,15]$. Furthermore, a nonempty set $P$ is called a polytope if it is a convex hull of a finite point set in $\mathbb{R}^{d}$. It is well known that polytopes can be characterized as bounded polyhedra. The main result of the paper is the following theorem.

Theorem 1.1 Let $d \in\{2,3\}$. Then for every $d$-dimensional polyhedron $P$ in $\mathbb{R}^{d}$, there exist d polynomials $p_{0}(x), \ldots, p_{d-1}(x) \in \mathbb{R}[x]$ with $P=\left(p_{0}, \ldots, p_{d-1}\right)_{\geq 0}$.

Bosse, Grötschel, and Henk [6] conjectured that, for every $d \geq 2, d$ polynomial inequalities suffice for representing a $d$-dimensional polytope. Theorem 1.1 confirms the above-mentioned conjecture for $d \leq 3$. The case where $d=2$ and $P$ is bounded is essentially due to Bernig; see [5]. For unbounded $P$, Theorem 1.1 is new for both $d=2$ and $d=3$. The conjecture was confirmed for simple polytopes of any dimension and a certain class of semi-algebraic sets containing all simple polytopes; see $[2,3]$. After completing the proof of Theorem 1.1, Ludwig Bröcker communicated to the authors a complete positive solution of the above-mentioned conjecture; cf. [7]. We point out that the arguments of Bröcker and our Proposition 7.3 also imply that all $d$-polyhedra (bounded and unbounded) can be described by $d$ polynomial inequalities.

We emphasize that the proof of Theorem 1.1 is constructive in the sense that we indicate explicit formulas for $p_{0}, \ldots, p_{d-1}$ (for more details, see also Sect. 2 below). However our construction has the undesirable property that the maximal degree of the polynomials $p_{0}, \ldots, p_{d-1}$ constructed in the proof depends not only on the combinatorial structure of $P$ (say, the number of facets and the dimension) but also on its metric structure (angles between faces and distances between vertices); furthermore we were also unable to find explicit upper bounds on the maximal degree of $p_{0}, \ldots, p_{d-1}$. It remains open whether Theorem 1.1 can be strengthened by requiring that the degrees of $p_{0}, \ldots, p_{d-1}$ are at most $n$ with $n$ depending only on the dimension and the number of facets of $P$. It should be noticed however that such bounds are possible if we allow somewhat more than $d$ polynomials in the representation of $P$. For example, as a consequence of the theorem of Bröcker and Scheiderer (which is valid for an arbitrary real closed field), in fact, one can show that, for every $d$-polytope $P$ in $\mathbb{R}^{d}$, there exist $p_{1}, \ldots, p_{d(d+1) / 2} \in \mathbb{R}[x]$ such that $P=\left(p_{1}, \ldots, p_{d(d+1) / 2}\right)_{\geq 0}$ and the degrees of $p_{1}, \ldots, p_{d(d+1) / 2}$ are at most $n$ with $n$ depending only on $d$ and the 
number of facets of $P$. This follows directly from the fact that the theorem of Bröcker and Scheiderer holds in an arbitrary real closed field (see [4, Chap. 1] for the definition of real closed fields) and from Theorem 5.1 in [13]. Analogously, if one could extend Theorem 1.1 to polytopes in an arbitrary real closed field, as a corollary, one would immediately get a strengthening of Theorem 1.1 with combinatorial bounds on the maximal degree of $p_{0}, \ldots, p_{d-1}$. Thus, we also ask whether Theorem 1.1 can be extended to arbitrary real closed fields.

The paper is organized as follows. Section 2 contains a sketch of the construction of the polynomials $p_{0}(x), \ldots, p_{d-1}(x)$ from Theorem 1.1 and indicates some proof ideas. Sections 3 and 4 present preliminaries from real algebraic geometry and convex geometry, respectively. In Sect. 5 we introduce and study a special family of neighborhoods of a given convex polytope, which is used in the main proof. Section 6 is concerned with approximation of polytopes and polyhedral cones by algebraic surfaces. In Sect. 7 we prove Theorem 1.1.

\section{A Sketch of the Construction}

We give a sketch of the construction of the polynomials $p_{0}(x), \ldots, p_{d-1}(x)$ from Theorem 1.1. Standard notions from convexity that are used in this section are introduced in Sect. 3 or can be found in the monographs [8, 14, 15].

Let $d \in\{2,3\}$ and $P$ be a $d$-dimensional polyhedron in $\mathbb{R}^{d}$. Assume that $P$ is bounded. Since the two-dimensional case was settled by Bernig [5], we may assume that $d=3$. Let $m$ be the number of facets of $P$. Then $P=\left\{x \in \mathbb{R}^{d}: q_{1}(x) \geq\right.$ $\left.0, \ldots, q_{m}(x) \geq 0\right\}$ for appropriate affine functions $q_{1}(x), \ldots, q_{m}(x)$. We define

$$
p_{2}(x):=q_{1}(x) \cdot \ldots \cdot q_{m}(x) .
$$

Further on, we construct a strictly concave polynomial $p_{0}(x)$ vanishing on each vertex of $P$ and such that the distance between $P$ and $\left(p_{0}\right)_{\geq 0}$ is small enough; see also Fig. $1^{1}$ With each vertex $v$ of $P$ we associate a polynomial $b_{v}(x)$ such that
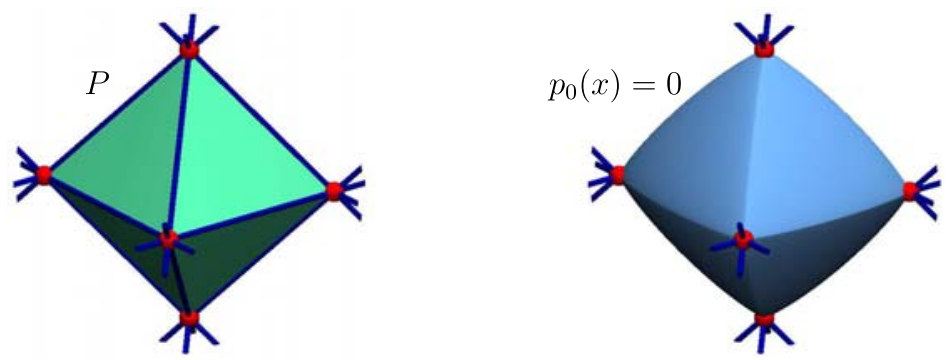

Fig. 1 Regular octahedron and an approximation of the octahedron by a strictly concave polynomial surface

\footnotetext{
${ }^{1}$ The figures in this subsection illustrate the construction for the case of the regular octahedron $\left\{\left(\xi_{1}, \xi_{2}, \xi_{3}\right) \in \mathbb{R}^{3}:\left|\xi_{1}\right|+\left|\xi_{2}\right|+\left|\xi_{3}\right| \leq 1\right\}$. Since octahedra are not simple polytopes, they are not covered by the main result of [2] on representation of simple polytopes by $d$ polynomial inequalities.
} 
Fig. 2 Polyhedron $P$ and the surface given by the equation $b_{v}(x)=0$

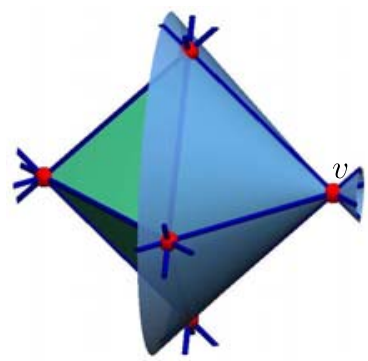

Fig. 3 The surface given by the equation $f_{v}(x)^{2 k} b_{v}(x)=0$

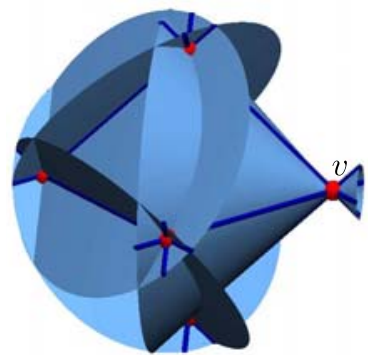

$\left(b_{v}\right)_{\geq 0}=B(v) \cup(2 v-B(v))$, where $B(v)$ is a pointed convex cone with apex at $v$ such that every edge of $P$ incident with $v$ lies in the boundary of $B(v)$; see Fig. 2. We define $p_{1}(x)$ as a "weighted combination" of the polynomials $b_{v}(x)$ with $v$ ranging over the set of all vertices of $P$. More precisely, we set

$$
p_{1}(x):=\sum_{v} f_{v}(x)^{2 k} b_{v}(x),
$$

where $v$ ranges over all vertices of $P, k \in \mathbb{N}$ is sufficiently large, and, for every vertex $v$ of $P, f_{v}(x)$ is the polynomial given by

$$
f_{v}(x):=\prod_{\substack{i=1, \ldots, m \\ q_{i}(v) \neq 0}} q_{i}(x),
$$

see also Fig. 3.

By construction $P \subseteq\left(p_{0}, p_{1}, p_{2}\right)_{\geq 0}$. We have $p_{0}(x)<0$ for all $x$ sufficiently far away from $P$. Further on, the region of all $x$ satisfying $p_{2}(x)<0$ is contiguous with all edges of $P$. Finally, if $G$ is a vertex or an edge of $P, x_{0}$ is a point in the relative interior of $G$, and $v$ and $w$ are two vertices of $P$ with $v \in G$ and $w \notin G$, respectively, then, for $x$ satisfying $p_{2}(x) \geq 0$ and converging to $x_{0}$, the term $f_{v}(x)^{2 k} b_{v}(x)$ dominates over $f_{w}(x)^{2 k} b_{w}(x)$, provided that $k$ is sufficiently large. The latter observation is used for analysis of the properties of $\left(p_{1}\right)_{\geq 0}$.

The geometry behind the construction is illustrated by the diagram in Fig. 4, where $P_{J}:=\left\{x \in \mathbb{R}^{3}: p_{j}(x) \geq 0 \forall j \in J\right\}$ for $J \subseteq\{1,2,3\}$ with $J \neq \emptyset$, and an arrow between two sets indicates the inclusion relation.

The case of unbounded $d$-polyhedra $P$ can be reduced to the case of bounded ones using some "homogenezation" idea; for details, see Sect. 7.2. 


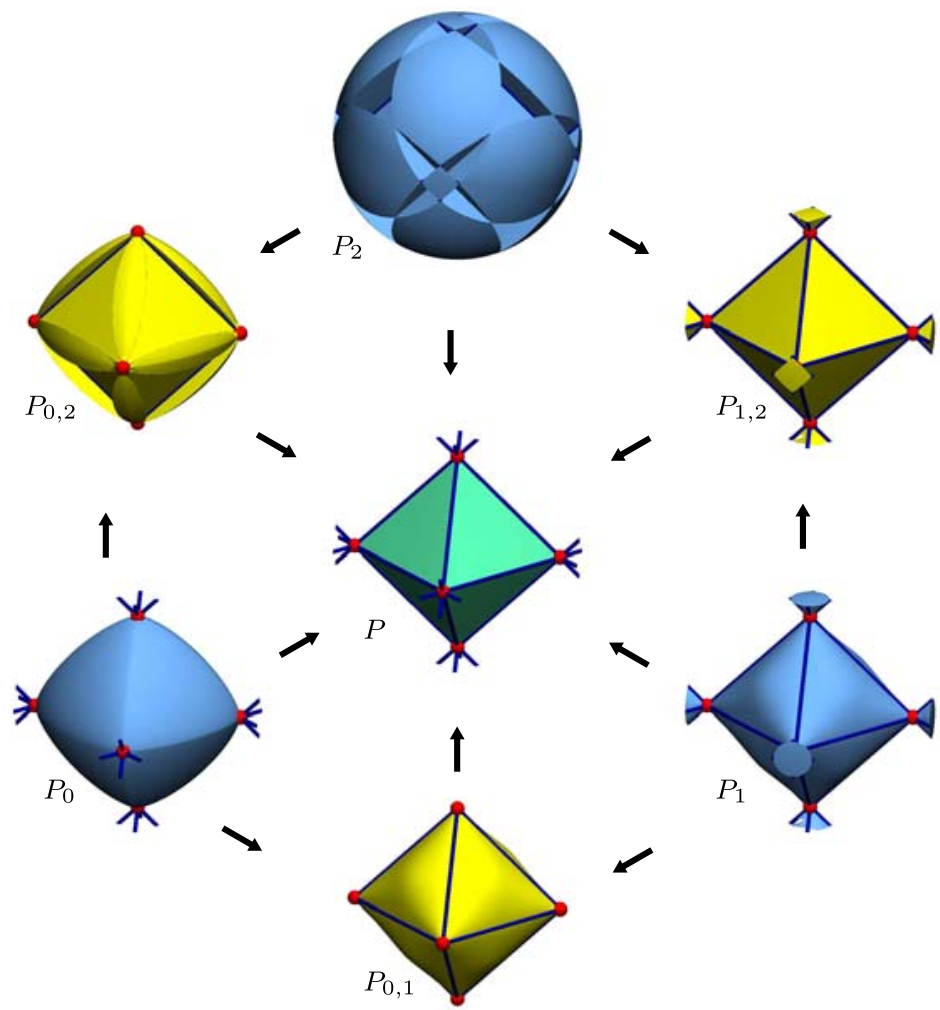

Fig. 4 Diagram illustrating representation of a three-dimensional polytope by three polynomial inequalities

\section{Preliminaries from Real Algebraic Geometry}

For information on real algebraic geometry and, in particular, the geometry of semialgebraic sets, we refer to $[1,4]$. If $x$ is a variable in $\mathbb{R}^{k}(k \in \mathbb{N})$, then $\mathbb{R}[x]$ stands for the class of $k$-variate polynomials over $\mathbb{R}$. Since $\mathbb{R}$ is a field of characteristic zero, we do not distinguish between real polynomials and real-valued polynomial functions. In particular, we can view affine functions as polynomials of degree at most one. A subset $A$ of $\mathbb{R}^{d}$ is said to be semi-algebraic if

$$
A=\bigcup_{i=1}^{n}\left\{x \in \mathbb{R}^{d}: f_{i, 1}(x)>0, \ldots, f_{i, s_{i}}(x)>0, g_{i}(x)=0\right\}
$$

for some $n, s_{1}, \ldots, s_{n} \in \mathbb{N}$ and $f_{i, j}(x), g_{i}(x) \in \mathbb{R}[x]$ with $i \in\{1, \ldots, n\}$ and $j \in$ $\left\{1, \ldots, s_{i}\right\}$.

A real-valued function $f(x)$ defined on a semi-algebraic set $A \subseteq \mathbb{R}^{d}$ is said to be a semi-algebraic function if the graph of $f(x)$ is a semi-algebraic subset of $\mathbb{R}^{d+1}$. The following theorem presents Łojasiewicz's Inequality; see [12], [4, Corollary 2.6.7]. 
Theorem 3.1 ([12]) Let $A$ be a bounded and closed semi-algebraic set in $\mathbb{R}^{d}$. Let $f(x)$ and $g(x)$ be continuous, semi-algebraic functions on A satisfying

$$
\{x \in A: f(x)=0\} \subseteq\{x \in A: g(x)=0\} .
$$

Then there exist $n \in \mathbb{N}$ and $\lambda>0$ such that $|g(x)|^{n} \leq \lambda|f(x)|$ for every $x \in A$.

\section{Preliminaries from Convexity}

For information on convex bodies and polytopes, we refer to [8, 14, 15]. The cardinality of a set is denoted by $|\cdot|$. The origin, Euclidean norm, and scalar product in $\mathbb{R}^{d}$ are denoted by $o,\|\cdot\|$, and $\langle\cdot, \cdot\rangle$, respectively. We endow $\mathbb{R}^{d}$ with its Euclidean topology. By $\mathbb{B}^{d}(c, \rho)$ we denote the closed Euclidean ball in $\mathbb{R}^{d}$ with center at $c \in \mathbb{R}^{d}$ and radius $\rho>0$. The notations aff, conv, int, and relint stand for the affine hull, convex hull, interior, and relative interior, respectively. If $X$ and $Y$ are nonempty sets in $\mathbb{R}^{d}$, we set $X \pm Y:=\{x \pm y: x \in X, y \in Y\}$. The Hausdorff distance $\operatorname{dist}(X, Y)$ between nonempty compact sets $X, Y \subseteq \mathbb{R}^{d}$ is defined by

$$
\operatorname{dist}(X, Y):=\min \left\{\rho \geq 0: X \subseteq Y+\mathbb{B}^{d}(o, \rho), Y \subseteq X+\mathbb{B}^{d}(o, \rho)\right\}
$$

see also [14, p. 48]. It is known that $\operatorname{dist}(X, Y)$ is a metric on the space of nonempty compacts sets in $\mathbb{R}^{d}$.

If for a convex set $X \subseteq \mathbb{R}^{d}$ and a point $x_{0} \in \mathbb{R}^{d}$, one has $x_{0}+\alpha\left(x-x_{0}\right) \in X$ for every $\alpha \geq 0$ and $x \in X$, then $X$ is said to be a convex cone with apex at $x_{0}$. Given a nonempty subset $X$ of $\mathbb{R}^{d}$, we introduce the conical hull cone $(X)$ of $X$ as the set of all possible linear combinations $\lambda_{1} x_{1}+\cdots+\lambda_{m} x_{m}$ with $m \in \mathbb{N}, \lambda_{i} \geq 0$, and $x_{i} \in X$ for $i \in\{1, \ldots, m\}$. Clearly, cone $(X)$ is a convex cone with apex at the origin.

The notions of polyhedron and polytope were defined in the introduction. Polyhedra (resp. polytopes) of dimension $n$ are referred to as $n$-polyhedra (resp. $n$-polytopes). A subset of $\mathbb{R}^{d}$ which is both a cone and polyhedron is said to be a polyhedral cone. A polyhedron is said to be line-free if it does not contain a straight line. The set $\operatorname{rec}(P):=\left\{u \in \mathbb{R}^{d}: P+u \subseteq P\right\}$ is said to be the recession cone of $P$. A line-free polyhedral cone is said to be pointed . Every polyhedron $P$ can be represented by

$$
P=Q+L+C,
$$

where $Q$ is a polytope, $L$ is a linear subspace of $\mathbb{R}^{d}$, and $C$ is a pointed polyhedral cone; see [15, Sect. 1.5]. For (4.1), one necessarily has $L+C=\operatorname{rec}(P)$. If $o \notin$ aff $P$, we introduce

$$
\operatorname{hom}(P):=\operatorname{cone}(P) \cup \operatorname{rec}(P) .
$$

It is known that $\operatorname{hom}(P)$ is a polyhedral cone; see [15, pp. 44-45]. Furthermore, it can be verified that if $P$ is line-free, then $\operatorname{hom}(P)$ is pointed.

For a convex polytope $P$ in $\mathbb{R}^{d}$, we introduce the support function and exposed face in direction $u$ by the equalities

$$
h(P, u):=\max \{\langle x, u\rangle: x \in P\} \quad \text { and } \quad F(P, u):=\{x \in P:\langle x, u\rangle=h(P, u)\},
$$


respectively. Given a polytope $P$ in $\mathbb{R}^{d}, \mathcal{F}(P)$ denotes the class of all faces of $P$, and, for $i \in\{-1, \ldots, d\}, \mathcal{F}_{i}(P)$ stands for the class of all $i$-faces of $P$ (i.e., faces of dimension $i$ ). We recall that, for every point $x$ of $P$, there exists a unique nonempty face $G$ of $P$ with $x \in$ relint $P$. Consequently, $P$ is the disjoint union of the relative interiors of all nonempty faces of $P$. A face $G$ of an $n$-polytope $P$ in $\mathbb{R}^{d}$ is said to be a facet of $P$ if $G$ has dimension $n-1$. If $G$ is a face of $P$, we define

$$
\mathcal{F}_{i}(G, P):=\left\{F \in \mathcal{F}_{i}(P): G \subseteq F\right\}
$$

By vert $(P)$ we denote the set of all vertices of $P$ (i.e., the set of 0 -dimensional faces). If $F$ is a facet of $P$, by $u_{F}(P)$ we denote the unit normal of $P$ at the facet $F$ which is parallel to $\operatorname{aff}(P)$ and satisfies $F\left(P, u_{F}(P)\right)=F$. More generally, if $G$ is a proper face of $P$, we define

$$
u_{G}(P):=\left(\sum_{F \in \mathcal{F}_{n-1}(G, P)} u_{F}(P)\right) /\left\|\sum_{F \in \mathcal{F}_{n-1}(G, P)} u_{F}(P)\right\|,
$$

where $n:=\operatorname{dim}(P)$. It is not hard to see that $F\left(P, u_{G}(P)\right)=G$.

With every facet $F$ of $P$ we associate the affine function

$$
q_{F}(P, x):=\frac{h\left(P, u_{F}(P)\right)-\left\langle u_{F}(P), x\right\rangle}{\operatorname{diam}(P)},
$$

where $\operatorname{diam}(P):=\max \{\|x-y\|: x, y \in P\}=\max \{\|x-y\|: x, y \in \operatorname{vert}(P)\}$. By construction, $0 \leq q_{F}(P, x)<1$ for every $x \in P$ with the equality $q_{F}(P, x)=0$ if and only if $x \in F$. For $v \in \operatorname{vert}(P)$, the supporting cone $S(P, v)$ of $P$ at $v$ is given by

$$
S(P, v):=\operatorname{cone}(P-v),
$$

see also $[14$, p. 70$]$. If $\operatorname{dim} P=d$, then

$$
S(P, v)=\left\{x \in \mathbb{R}^{d}:\left\langle u_{F}(P), x\right\rangle \leq 0 \forall F \in \mathcal{F}_{d-1}(v, P)\right\}
$$

and by this

$$
v+S(P, v)=\left\{x \in \mathbb{R}^{d}: q_{F}(P, x) \leq 0 \forall F \in \mathcal{F}_{d-1}(v, P)\right\} .
$$

For every $v \in \operatorname{vert}(P)$, we define the hyperplane

$$
H_{v}(P):=\left\{x \in \mathbb{R}^{d}:\left\langle x, u_{v}(P)\right\rangle=-1\right\}
$$

and the polytope

$$
P_{v}:=S(P, v) \cap H_{v}(P) .
$$

It can be shown that cone $\left(P_{v}\right)=S(P, v)$. Hence the cone

$$
S_{\rho}(P, v):=\operatorname{cone}\left(\left(P_{v}+\mathbb{B}^{d}(o, \rho)\right) \cap H_{v}(P)\right)
$$


with $\rho>0$ and $v \in \operatorname{vert}(P)$ can be viewed as an "outer approximation" of the supporting cone with the parameter $\rho$ controlling the quality of the approximation. For every $v \in \operatorname{vert}(P)$, one has

$$
P \backslash\{v\} \subseteq \operatorname{int}\left(v+S_{\rho}(P, v)\right) .
$$

For a polytope $P$ in $\mathbb{R}^{d}$ and $x \in$ aff $P$, we introduce the notation

$$
\mathcal{F}^{-}(P, x):=\left\{F \in \mathcal{F}_{d-1}(P): q_{F}(P, x) \leq 0\right\} .
$$

The class $\mathcal{F}^{-}(P, x)$ be interpreted as the set of all facets of the polytope $P$ which are visible from $x$.

In Theorem 1.1 and the remaining statements dealing with algorithms, a polyhedron is assumed to be given by a system of affine inequalities (the so-called $\mathrm{H}$ representation ), and a polynomial by a list of its coefficients. It is not difficult to show that there exists an algorithm that takes a polytope $P$ and constructs all functions $q_{F}(P, x)$ where $F$ is a facet of $P$.

\section{A Family of Neighborhoods of a 3-polytope}

Let $\varepsilon>0, \rho>0$, and $P$ be a 3-polytope in $\mathbb{R}^{3}$. We set $U_{P}(P, \varepsilon, \rho):=P$ and $U_{F}(P, \varepsilon, \rho):=\left\{x \in \mathbb{R}^{3}: \mathcal{F}^{-}(P, x)=\{F\}\right\}$ for $F \in \mathcal{F}_{2}(P)$. Furthermore, for $I \in$ $\mathcal{F}_{1}(P)$ and $v \in \operatorname{vert}(P)$, we define

$$
\begin{aligned}
U_{I}(P, \varepsilon, \rho):= & \left(I+\mathbb{B}^{3}(o, \varepsilon)\right) \cap\left\{x \in \mathbb{R}^{3}: \mathcal{F}_{2}(I, P) \subseteq \mathcal{F}^{-}(P, x)\right\} \\
& \cap\left(\bigcap_{v \in \operatorname{vert}(P)} S_{\rho}(P, v)\right), \\
U_{v}(P, \varepsilon, \rho):= & \mathbb{B}^{3}(v, \varepsilon) \backslash\left(v+\operatorname{int} S_{\rho}(P, v)\right),
\end{aligned}
$$

see also Figs. 5 and 6. We also introduce the region

$$
U_{v}^{\prime}(P, \varepsilon, \rho):=\mathbb{B}^{3}(v, \varepsilon) \backslash\left(\left(v+\operatorname{int} S_{\rho}(P, v)\right) \cup\left(v-\operatorname{int} S_{\rho}(P, v)\right)\right),
$$

which is a subset of $U_{v}(P, \varepsilon, \rho)$; see Fig. 7. With $P$ we associate the set

$$
U(P, \varepsilon, \rho):=\bigcup_{G \in \mathcal{F}(P) \backslash\{\emptyset\}} U_{G}(P, \varepsilon, \rho) .
$$

Fig. 5 The regions $U_{I}(P, \varepsilon, \rho)$ with $I \in \mathcal{F}_{1}(P)$

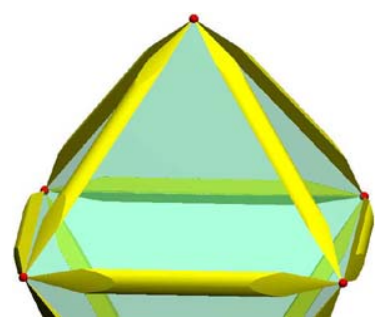


Fig. 6 The regions $U_{v}(P, \varepsilon, \rho)$ with $v \in \operatorname{vert}(P)$

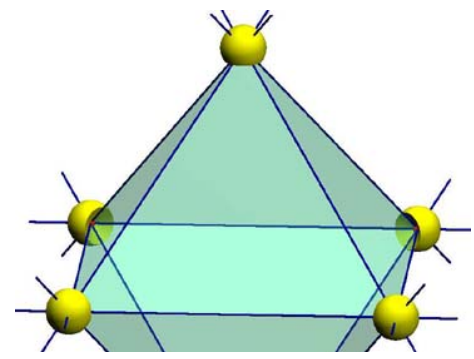

Fig. 7 The regions $U_{v}^{\prime}(P, \varepsilon, \rho)$ with $v \in \operatorname{vert}(P)$

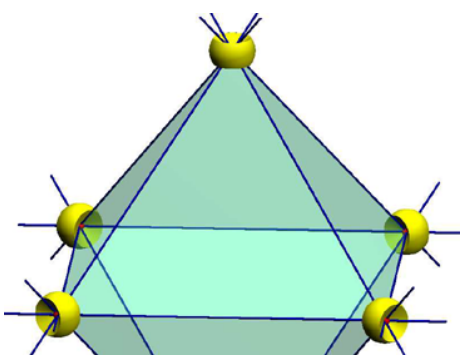

The main statement of this section is Proposition 5.1, whose formulation is intuitively clear. However, we are not aware of any short proof of it.

Proposition 5.1 Let $P$ be a 3-polytope in $\mathbb{R}^{3}$. Then $P \subseteq \operatorname{int} U(P, \varepsilon, \rho)$ for all sufficiently small $\rho>0$ and all $\varepsilon>0$.

The proof of Proposition 5.1 is based on a number of auxiliary statements. The proofs of simple Lemmas 5.2, 5.3, and 5.4 are omitted.

Lemma 5.2 Let $P$ be a d-polytope in $\mathbb{R}^{d}, G$ a nonempty face of $P$, and $x \in$ relint $G$. Then there exists $\varepsilon>0$ such that every $y \in \mathbb{B}^{d}(x, \varepsilon)$ satisfies $\mathcal{F}^{-}(P, y) \subseteq$ $\mathcal{F}_{d-1}(G, P)$

If a point $x$ outside $P$ is sufficiently close to $P$, then all facets of $P$ visible from $x$ have a vertex in common. This is stated in a more formal way in the following lemma.

Lemma 5.3 Let $P$ be a d-polytope in $\mathbb{R}^{d}$. Then for some $\varepsilon>0$ and every $x \in P+$ $\mathbb{B}^{d}(o, \varepsilon)$, there exists $v \in \operatorname{vert}(P)$ satisfying $\mathcal{F}^{-}(P, x) \subseteq \mathcal{F}_{d-1}(v, P)$.

Lemma 5.4 Let $P$ be a d-polytope in $\mathbb{R}^{d}$ with $o \in \operatorname{vert}(P)$, and let $F \in \mathcal{F}_{d-1}(o, P)$. Then $G:=\operatorname{aff} F \cap P_{o}$ is a facet of $P_{o}$. Furthermore, for some $\alpha>0$, one has

$$
q_{F}(P, x)=\alpha \cdot q_{G}\left(P_{o}, x\right) \quad \forall x \in H_{o}(P) .
$$


Lemma 5.5 Let $P$ be a 3-polytope in $\mathbb{R}^{3}$. Then there exists $\rho>0$ such that, for all $v \in \operatorname{vert}(P)$ and $x \in\left(v+S_{\rho}(P, v)\right) \backslash\{v\}$, one can find $I \in \mathcal{F}_{1}(v, P)$ satisfying

$$
\mathcal{F}^{-}(P, x) \cap \mathcal{F}_{2}(v, P) \subseteq \mathcal{F}_{2}(I, P) .
$$

Proof Consider an arbitrary $v \in \operatorname{vert}(P)$. Replacing $P$ by an appropriate translation, we assume that $v=o$. By Lemma 5.3 applied to $P_{o}$, one can choose $\rho>0$ such that, for every $x \in H_{o}(P) \cap\left(P_{o}+\mathbb{B}^{3}(o, \rho)\right)$, there exists $w \in \operatorname{vert}\left(P_{o}\right)$ satisfying $\mathcal{F}^{-}\left(P_{o}, x\right) \subseteq \mathcal{F}_{1}\left(w, P_{o}\right)$. Take an arbitrary $x \in S_{\rho}(P, o) \backslash\{o\}$. Then $\left\langle x, u_{o}(P)\right\rangle<0$, and we introduce

$$
y:=-\frac{x}{\left\langle x, u_{o}(P)\right\rangle} \in H_{o}(P) \cap\left(P_{o}+\mathbb{B}^{3}(o, \rho)\right) .
$$

By the choice of $\rho$, there exists $w \in \operatorname{vert}\left(P_{v}\right)$ with $\mathcal{F}^{-}\left(P_{o}, y\right) \subseteq \mathcal{F}_{1}\left(w, P_{o}\right)$. In view of Lemma 5.4, the latter inclusion implies $\mathcal{F}^{-}(P, x) \cap \mathcal{F}_{2}(o, P) \subseteq \mathcal{F}_{2}(I, P)$, where $I \in \mathcal{F}_{1}(o, P)$ is such that $H_{o}(P) \cap \operatorname{cone}(I)=\{w\}$. This shows (5.3).

Now we are ready to prove the main statement of this section.

Proof of Proposition 5.1 We pick an arbitrary $x \in P$ and show that $x \in \operatorname{int} U(P, \varepsilon, \rho)$. By $G$ we denote the unique face of $P$ with $x \in \operatorname{relint} G$. If $G=P$, then $x \in$ int $P$, and by this $x \in \operatorname{int} U(P, \varepsilon, \rho)$. If $G$ is a facet of $P$, then, in view of Lemma 5.2, all points sufficiently close to $x$ lie in $P \cup U_{G}(P, \varepsilon, \rho)$. If $G$ is an edge of $P$, we show that

$$
x \in \operatorname{int}\left(P \cup\left(\bigcup_{F \in \mathcal{F}_{2}(G, P)} U_{F}(P, \varepsilon, \rho)\right) \cup U_{G}(P, \varepsilon, \rho)\right) .
$$

In fact, it is easy to see that, for every $v \in \operatorname{vert}(P)$, one has $x \in v+\operatorname{int} S_{\rho}(P, v)$ and $x \in \operatorname{int}\left(G+\mathbb{B}^{3}(o, \varepsilon)\right)$. Furthermore, applying Lemma 5.2, we obtain

$$
x \in \operatorname{int}\left\{y \in \mathbb{R}^{3}: \mathcal{F}^{-}(F, y) \subseteq \mathcal{F}_{2}(G, P)\right\} .
$$

Thus, (5.4) is fulfilled, and by this $x \in \operatorname{int} U(P, \varepsilon, \rho)$.

It remains to consider the case $x=v$ for some $v \in \operatorname{vert}(P)$. We assume that $\rho$ is small enough so that the assertion of Lemma 5.5 is fulfilled. We show that $x \in$ int $U(P, \varepsilon, \rho)$ by contradiction. Assume that there exists a sequence $\left(x_{n}\right)_{n=1}^{\infty}$ converging to $x$ and such that $x_{n} \notin U(P, \varepsilon, \rho)$ for every $n \in \mathbb{N}$. Take an arbitrary $\mathcal{F}^{*} \subseteq$ $\mathcal{F}_{2}(P)$ such that $\mathbb{N}^{*}:=\left\{n \in \mathbb{N}: \mathcal{F}^{-}\left(P, x_{n}\right)=\mathcal{F}^{*}\right\}$ is infinite. By Lemma 5.2, for all sufficiently large $n \in \mathbb{N}$, one has $\mathcal{F}^{-}\left(P, x_{n}\right) \subseteq \mathcal{F}_{2}(v, P)$. Hence $\mathcal{F}^{*} \subseteq \mathcal{F}_{2}(v, P)$. If $\left|\mathcal{F}^{*}\right|=1$, then $x_{n} \in U(P, \varepsilon, \rho)$ for every $n \in \mathbb{N}^{*}$. If $\left|\mathcal{F}^{*}\right| \geq 3$, then for all sufficiently large $n$, one has $x_{n} \notin v+S_{\rho}(P, v)$, and by this $x_{n} \in \mathbb{B}^{3}(v, \varepsilon) \backslash(v+$ int $\left.S_{\rho}(P, v)\right)=U_{v}(\varepsilon, \rho)$. In fact, if we assume the contrary, i.e., $x_{n} \in v+S_{\rho}(P, v)$ for all sufficiently large $n \in \mathbb{N}^{*}$, then, by (5.3), we see that for some $I \in \mathcal{F}_{1}(v, P)$, one has $\mathcal{F}^{*} \cap \mathcal{F}_{2}(v, P) \subseteq \mathcal{F}_{2}(I, P)$. In view of $\mathcal{F}^{*} \subseteq \mathcal{F}_{2}(v, P)$, the latter implies $\mathcal{F}^{*} \subseteq \mathcal{F}_{2}(I, P)$. Hence $\left|\mathcal{F}^{*}\right| \leq 2$, a contradiction. We consider the remaining case $\left|\mathcal{F}^{*}\right|=2$. In view of (4.2), one has $x_{n} \in S_{\rho}(P, w)$ for all sufficiently large $n$ and 
every $w \in \operatorname{vert}(P) \backslash\{v\}$. It is easy to see that, for all sufficiently large $n$, one has $x_{n} \in \mathbb{B}^{3}(v, \varepsilon)$. Hence, for all large $n$, one has $x_{n} \in U_{v}(P, \varepsilon, \rho)$ if $x \notin v+$ int $S_{\rho}(P, v)$, and, taking into account the assertion of Lemma 5.5 and the definition of $U_{I}(P, \varepsilon, \rho)$, one has $x_{n} \in U_{I}(P, \varepsilon, \rho)$ for some $I \in \mathcal{F}_{1}(v, P)$, provided that $x \in v+S_{\rho}(P, v)$. Consequently, for all large $n$, one has $x_{n} \in U(P, \varepsilon, \rho)$. This yields the assertion of the proposition.

\section{Approximation of Polytopes and Polyhedral Cones}

A polynomial $f \in \mathbb{R}[x]$ of degree $n$ is homogeneous if $f(\alpha x)=\alpha^{n} f(x)$ for all $\alpha \in \mathbb{R}$ and $x \in \mathbb{R}^{d}$. Consider a hyperplane $H$ in $\mathbb{R}^{d}$ with $o \notin H$. Then

$$
H=\left\{x \in \mathbb{R}^{d}:\langle x, u\rangle=1\right\}
$$

with $u \in \mathbb{R}^{d} \backslash\{o\}$ uniquely determined by $H$. Consider a polynomial $f \in \mathbb{R}[x]$ of degree $n$. The homogeneous continuation of $\left.f(x)\right|_{H}$ (i.e., the restriction of $f(x)$ to $H$ ) to $\mathbb{R}^{d}$ is defined as the polynomial $\tilde{f} \in \mathbb{R}[x]$ uniquely determined by $\tilde{f}(x)=$ $\langle x, u\rangle^{n} f(x /\langle x, u\rangle)$ with $x \in \mathbb{R}^{d} \backslash H$. Clearly, $\tilde{f}$ is a homogeneous polynomial.

We say that there is an algorithm that constructs a sequence $\left(p_{l}(x)\right)_{l=1}^{\infty}$ of polynomials from the given input if there exists an algorithm which constructs $p_{l}(x)$ from $l \in \mathbb{N}$ and the given input. Theorem 6.1 and Proposition 6.3 are the main statements of this section. The former is concerned with a special type of approximation of polytopes, and the latter with approximation of supporting cones of polytopes. Given a polytope $P$ in $\mathbb{R}^{d}$, we introduce the following conditions on a sequence $\left(g_{l}(x)\right)_{l=1}^{\infty}$ of polynomials from $\mathbb{R}[x]$.

$\mathcal{A}(P)$ : The Hausdorff distance from $P$ to $\left\{x \in\right.$ aff $\left.P: g_{l}(x) \geq 0\right\}$ converges to zero as $l \rightarrow \infty$.

$\mathcal{I}(P)$ : For every $v \in \operatorname{vert}(P)$ and $l \in \mathbb{N}$, one has $g_{l}(v)=0$.

$\mathcal{C}(P)$ : For every $l \in \mathbb{N}$, the function $\left.g_{l}(x)\right|_{\text {aff } P}$ is strictly concave.

$\mathcal{H}(P)$ : For every $l \in \mathbb{N}$, one has

$$
\left\{x \in \mathbb{R}^{d}: \tilde{g}_{l}(x) \geq 0,\langle x, u\rangle \geq 0\right\}=\operatorname{cone}\left(\left\{x \in \text { aff } P: g_{l}(x) \geq 0\right\}\right),
$$

where $\tilde{g}_{l}(x)$ is a homogeneous continuation of $\left.g_{l}(x)\right|_{\text {aff } P}$.

The notations $\mathcal{A}, \mathcal{I}, \mathcal{C}$, and $\mathcal{H}$ are derived from the words "approximation", "interpolation", "concavity", and "homogeneity", respectively. Condition $\mathcal{H}(P)$ makes sense only for the case where $o \notin$ aff $P$.

Theorem 6.1 Let $P$ be a polytope in $\mathbb{R}^{d}$. Then the following statements hold true.

I. There exists a sequence $\left(g_{l}(x)\right)_{l=1}^{\infty}$ of polynomials from $\mathbb{R}[x]$ that satisfy $\mathcal{A}(P)$, $\mathcal{I}(P), \mathcal{C}(P)$.

II. For every sequence $\left(g_{l}(x)\right)_{l=1}^{\infty}$ satisfying $\mathcal{A}(P), \mathcal{I}(P), \mathcal{C}(P)$, there exists $\rho>0$ with

$$
\left(v-S_{\rho}(P, v)\right) \cap\left\{x \in \operatorname{aff} P: g_{l}(P, x) \geq 0\right\}=\{v\} \quad \forall v \in \operatorname{vert}(P) \forall l \in \mathbb{N} .
$$


III. If $\operatorname{dim}(P)=d-1$ and $o \notin$ aff $P$, there exists a sequence $\left(g_{l}(x)\right)_{l=1}^{\infty}$ of polynomials from $\mathbb{R}[x]$ that satisfy $\mathcal{A}(P), \mathcal{I}(P), \mathcal{C}(P), \mathcal{H}(P)$.

Proof Let $n:=\operatorname{dim}(P)$.

Part I. For $l \in \mathbb{N}$, we define $g_{l}(x)$ by

$$
g_{l}(x):=1-\sum_{v \in \operatorname{vert}(P)} y_{v, l}\left(\frac{1}{\left|\mathcal{F}_{n-1}(v, P)\right|} \sum_{F \in \mathcal{F}_{n-1}(v, P)}\left(1-q_{F}(P, x)\right)^{2\left(l+l_{0}\right)}\right)^{2\left(l+l_{0}\right)},
$$

where $l_{0} \in \mathbb{N}$ and $y_{v, l} \in \mathbb{R}$. In [2] it was shown that an appropriate $l_{0} \in \mathbb{N}$ and scalars $y_{v, l}$ with $v \in \operatorname{vert}(P)$ and $l \in \mathbb{N}$ can be constructed such that the sequence $\left(g_{l}(x)\right)_{l=1}^{\infty}$ satisfies $\mathcal{I}(P)$ an $\mathcal{A}(P)$. By construction, $\left(g_{l}(x)\right)_{l=1}^{\infty}$ also satisfies condition $\mathcal{C}(P)$.

Part II. Without loss of generality we may assume that $n=d$. Consider an arbitrary $v \in \operatorname{vert}(P)$. Replacing $P$ by an appropriate translation, we assume that $v=o$. Let $\rho_{o}^{\prime}:=\min \left\{\|x-y\|: x \in P, y \in\left(-P_{o}\right)\right\}$. We show that there exists $l_{o}^{\prime} \in \mathbb{N}$ such that

$$
\forall l \geq l_{o}^{\prime} \forall x \in\left(-P_{o}+\mathbb{B}^{d}\left(o, \rho_{o}^{\prime} / 2\right)\right) \cap\left(-H_{o}(P)\right): \quad g_{l}(x)<0 .
$$

Assume the contrary. Then there exist sequences $\left(l_{k}\right)_{k=1}^{\infty},\left(x_{k}\right)_{k=1}^{\infty}$, and $\left(y_{k}\right)_{k=1}^{\infty}$ such that $l_{k} \in \mathbb{N}, x_{k} \in\left(-H_{o}(P)\right), y_{k} \in-P_{o},\left\|x_{k}-y_{k}\right\| \leq \rho_{o}^{\prime} / 2$, and $g_{l_{k}}\left(x_{k}\right) \geq 0$ for every $k \in \mathbb{N}$. Then, for every $k \in \mathbb{N}$, we obtain

$$
\begin{aligned}
& \operatorname{dist}\left(P,\left\{x \in \operatorname{aff} P: g_{l_{k}}(x) \geq 0\right\}\right) \\
& \quad \geq \min _{x \in P}\left\|x-x_{k}\right\| \geq \min _{x \in P}\left\|x-y_{k}\right\|-\left\|x_{k}-y_{k}\right\| \\
& \geq \rho_{o}^{\prime}-\left\|x_{k}-y_{k}\right\| \geq \rho_{o}^{\prime} / 2>0,
\end{aligned}
$$

a contradiction to the assumption that $\left(g_{l}(x)\right)_{l=1}^{\infty}$ satisfies $\mathcal{A}(P)$. Let us fix $l_{o}^{\prime}$ satisfying (6.4). Next we show that, for every $l \in \mathbb{N}$ and every $x \in-P_{o}$, one has $g_{l}(x)<0$. Assume the contrary, i.e., $g_{l}(x) \geq 0$. Since $\left(g_{l}(x)\right)_{l=1}^{\infty}$ satisfies $\mathcal{C}(P)$ and $\mathcal{I}(P)$, we deduce that $g_{l}(y)>0$ for every $y \in P \backslash \operatorname{vert}(P)$. For a sufficiently small $\varepsilon>0$, one has $-\varepsilon x \in P \backslash \operatorname{vert}(P)$. Then $o$ is a convex combination of $-\varepsilon x$ and $y$ (with nonzero coefficients). Hence, from the strict convexity of $g_{l}(x)$ and from $g_{l}(-\varepsilon x)>0$ and $g_{l}(x) \geq 0$ we deduce that $g_{l}(o)>0$, a contradiction. Hence $g_{l}(x)<0$ for every $l \in \mathbb{N}$ and every $x \in-P_{o}$, and therefore we may fix $\rho_{o, l}>0$ such that $\left(-P_{o}+\mathbb{B}^{d}\left(o, \rho_{o, l}^{\prime}\right)\right) \cap\left(-H_{o}\right)$ is disjoint with $\left\{x \in\right.$ aff $\left.P: g_{l_{k}}(x) \geq 0\right\}$. Applying the above arguments to all vertices of $P$, we determine quantities $\rho_{v, l}$ and $\rho_{v}^{\prime}$ with $v \in \operatorname{vert}(P)$ and $l \in \mathbb{N}$.

The assertion follows by setting

$$
l^{\prime}:=\max \left\{l_{v}^{\prime}: v \in \operatorname{vert}(P)\right\}, \quad \rho_{v}:=\min \left\{\rho_{v, 1}, \ldots, \rho_{v, l^{\prime}}, \rho_{v}^{\prime} / 2\right\}
$$

for every $v \in \operatorname{vert}(P)$ and taking $\rho:=\min \left\{\rho_{v}: v \in \operatorname{vert}(P)\right\}$.

Part III. We assume that $o \notin$ aff $P$. Without loss of generality we also assume that $n=d-1$. For $l \in \mathbb{N}$, the homogeneous continuation $\tilde{g}_{l}(x)$ of $\left.g_{l}(x)\right|_{\text {aff } P}$ can be 
expressed by

$$
\begin{aligned}
\tilde{g}_{l}(x):= & \langle x, u\rangle^{4\left(l+l_{0}\right)^{2}}-\sum_{v \in \operatorname{vert}(P)} y_{v, l}\left(\frac{1}{\left|\mathcal{F}_{d-2}(v, P)\right|}\right. \\
& \left.\times \sum_{F \in \mathcal{F}_{d-2}(v, P)}\left(\langle x, u\rangle-\tilde{q}_{F}(P, x)\right)^{2\left(l+l_{0}\right)}\right)^{2\left(l+l_{0}\right)},
\end{aligned}
$$

where $\tilde{q}_{F}(P, x)$ is a homogeneous continuation of $\left.q_{F}(P, x)\right|_{\text {aff } P}$, i.e.,

$$
\tilde{q}_{F}(P, x):=\frac{h\left(P, u_{F}(P)\right)\langle u, x\rangle-\left\langle u_{F}(P), x\right\rangle}{\operatorname{diam}(P)} .
$$

If $\langle x, u\rangle=0$, then (6.5) amounts to

$$
\tilde{g}_{l}(x):=-\sum_{v \in \operatorname{vert}(P)} y_{v, k}\left(\frac{1}{\left|\mathcal{F}_{d-2}(v, P)\right|} \sum_{F \in \mathcal{F}_{d-2}(v, P)}\left(\frac{\left\langle u_{F}(P), x\right\rangle}{\operatorname{diam}(P)}\right)^{2\left(l+l_{0}\right)}\right)^{2\left(l+l_{0}\right)} .
$$

Thus, if $\langle x, u\rangle=0$, we have $\tilde{g}_{l}(x) \leq 0$ with equality if and only if $x=o$. Directly from the definition of homogeneous continuation it follows

$$
\left\{x \in \mathbb{R}^{d}:\langle x, u\rangle>0, \tilde{g}_{l}(x) \geq 0\right\} \cup\{o\}=\operatorname{cone}\left(\left\{x \in H: g_{l}(x) \geq 0\right\}\right) .
$$

Equalities (6.6) and (6.7) yield the assertion.

We shall need the following observation.

Lemma 6.2 The degree of every nonzero concave polynomial in $\mathbb{R}[x]$ is even.

Proposition 6.3 Let $P$ be a 3-polytope in $\mathbb{R}^{3}$, and let $v \in \operatorname{vert}(P)$. Then there exists a sequence of polynomials $\left(b_{v, l}(x)\right)_{l=1}^{\infty}$ satisfying the following conditions:

I. For every $l \in \mathbb{N}$, the polynomial $b_{v, l}(x)$ is of even degree

II. For every $l \in \mathbb{N}$, the polynomial $b_{v, l}(x+v)$ is homogeneous.

III. For all $\rho>0, l \in \mathbb{N}, I \in \mathcal{F}_{1}(v, P)$, and $x \in v+S_{\rho}(P, v)$ satisfying $\mathcal{F}^{-}(P, x)=$ $\mathcal{F}_{2}(I, P)$, one has $b_{v, l}(x) \leq 0$ with equality if and only if $x \in \operatorname{cone}(I-v)+v$.

IV. For every $l \in \mathbb{N}$, the set

$$
B_{l}(P, v):=\left\{x \in \mathbb{R}^{3}:\left\langle x-v, u_{v}(P)\right\rangle \leq 0, b_{v, l}(x) \geq 0\right\}
$$

is a convex cone which has apex at $v$ and satisfies $P \subseteq B_{l}(P, v)$.

V. For every $\rho>0$,

$$
\begin{gathered}
(v+S(P, v)) \cup(v-S(P, v)) \subseteq\left(b_{v, l}\right)_{\geq 0} \subseteq\{v\} \\
\cup\left(v+\operatorname{int} S_{\rho}(P, v)\right) \cup\left(v-\operatorname{int} S_{\rho}(P, v)\right)
\end{gathered}
$$

if $l \in \mathbb{N}$ is sufficiently large. 
Proof Replacing $P$ by an appropriate translation, we may assume that $v=o$. Applying Theorem 6.1(III), we determine a sequence $\left(g_{o, l}(x)\right)_{l=1}^{\infty}$ which satisfies $\mathcal{A}\left(P_{o}\right)$, $\mathcal{I}\left(P_{o}\right)$, and $\mathcal{C}\left(P_{o}\right)$, respectively. We define $b_{o, l}(x):=\tilde{g}_{o, l}(x-v)$, where $\tilde{g}_{o, l}(x)$ is the homogeneous continuation of $\left.g_{o, l}(x)\right|_{H_{o}(P)}$.

The part of Statement I follows Lemma 6.2. Statements II follows by construction.

Let us show Statement III. Consider an arbitrary $\rho>0$ and $x \in S_{\rho}(P, v)$ with $\mathcal{F}^{-}(P, x)=\mathcal{F}_{2}(I, P)$. From the definition of $S_{\rho}(P, v)$ it follows that $\left\langle x, u_{o}(P)\right\rangle<0$, and by this $y:=\frac{x}{\left|\left\langle x, u_{o}(P)\right\rangle\right|} \in H_{o}(P)$. The intersection of cone $(I)$ and $H_{o}(P)$ is a vertex of $P_{o}$, which we denote by $w$. Since $\mathcal{F}^{-}(P, x)=\mathcal{F}_{2}(I, P)$, taking into account Lemma 5.4, we obtain $\mathcal{F}^{-}\left(P_{o}, y\right)=\mathcal{F}_{2}\left(w, P_{o}\right)$. Consequently, $y \in w-S\left(P_{o}, w\right)$. Then, by Theorem 6.1 (II), we get $g_{o, l}(y) \leq 0$ with equality if and only if $y=w$. Consequently, $\tilde{g}_{o, l}(x) \leq 0$ with equality if and only if $x \in S_{\rho}(P, o) \cap$ aff $I=\operatorname{cone}(I)$.

Statement IV follows directly from Theorem 6.1(III).

It remains to show Statement V. Consider an arbitrary $\rho>0$. Since $\left(g_{o, l}(x)\right)_{l=1}^{\infty}$ satisfies $\mathcal{I}\left(P_{o}\right)$ and $\mathcal{C}\left(P_{o}\right)$, in view of the definition of $b_{o, l}(x)$, we obtain

$$
S(P, o) \cup(-S(P, o)) \subseteq\left(b_{o, l}\right)_{\geq 0} .
$$

By construction, $b_{o, l}(x)=b_{o, l}(-x)$ for every $x \in \mathbb{R}^{3}$. Consequently, $\left(b_{o, l}\right)_{\geq 0}=$ $B_{l}(P, o) \cup\left(-B_{l}(P, o)\right)$. Since $\left(g_{o, l}(x)\right)_{l=1}^{\infty}$ satisfies $\mathcal{A}\left(P_{o}\right), \mathcal{I}\left(P_{o}\right)$, and $\mathcal{C}\left(P_{o}\right)$, if $l$ is large enough, the relation

$$
\left(b_{o, l}\right)_{\geq 0} \subseteq S_{\rho / 2}(P, o) \cup\left(-S_{\rho / 2}(P, o)\right)
$$

holds true. Obviously,

$$
S_{\rho / 2}(P, o) \cup\left(-S_{\rho / 2}(P, o)\right) \subseteq\{o\} \cup \operatorname{int} S_{\rho}(P, o) \cup\left(-\operatorname{int} S_{\rho}(P, o)\right),
$$

and we arrive at (6.8).

\section{Proof of the Main Result}

\subsection{The Case of Bounded Polyhedra}

The following theorem implies Theorem 1.1 for the case where $P$ is a $d$-polytope with $d \leq 3$. (As mentioned in the introduction, the case $d=2$ is due to Bernig.)

Theorem 7.1 Let $d \in\{2,3\}$ and $P$ be a d-dimensional polytope in $\mathbb{R}^{d}$. Let $\left(g_{m}(x)\right)_{m=1}^{\infty}$ be a sequence of polynomials satisfying conditions $\mathcal{A}(P), \mathcal{I}(P)$, $\mathcal{C}(P)$. Then there exist polynomials $p_{1}(x), \ldots, p_{d-1}(x) \in \mathbb{R}[x]$ such that $P=$ $\left(p_{0}, \ldots, p_{d-1}\right)_{\geq 0}$ and

$$
p_{0}(x)=g_{m}(x)
$$

for some $m \in \mathbb{N}$. 
Proof We consider the case $d=3$. First, we show the existence of polynomials $p_{0}(x), p_{1}(x), p_{2}(x)$ satisfying $P=\left(p_{0}, p_{1}, p_{2}\right)_{\geq 0}$, and then we show that these polynomials are constructible. Obviously, there exists $\varepsilon_{0}>0$ such that

$$
\forall F \in \mathcal{F}_{d-1}(P) \forall G \in \mathcal{F}(P): \quad F \cap G=\emptyset \Longrightarrow \text { aff } F \cap\left(G+\mathbb{B}^{d}\left(o, \varepsilon_{0}\right)\right)=\emptyset .
$$

In view of Theorem 6.1(II), condition (6.2) is fulfilled for all sufficiently small $\rho>0$. We also assume that $\rho$ is sufficiently small so that (5.3) in Lemma 5.5 is fulfilled. For $v \in \operatorname{vert}(P)$ and $l \in \mathbb{N}$, let the polynomials $b_{v, l}(x)$ be as in Proposition 6.3. Fix $\alpha_{l}>0$ satisfying

$$
\left|b_{w, l}(x)\right| \leq \alpha_{l} \quad \forall x \in P+\mathbb{B}^{3}\left(o, \varepsilon_{0}\right) \forall w \in \operatorname{vert}(P) .
$$

We define

$$
\begin{aligned}
& p_{0}(x):=g_{m}(x), \\
& p_{1}(x):=\sum_{v \in \operatorname{vert}(P)} f_{v}(x)^{2 k} b_{v, l}(x), \\
& p_{2}(x):=\prod_{F \in \mathcal{F}_{2}(P)} q_{F}(P, x),
\end{aligned}
$$

where

$$
f_{v}(x):=\prod_{F \in \mathcal{F}_{2}(P) \backslash \mathcal{F}_{2}(v, P)} q_{F}(P, x)
$$

for every $v \in \operatorname{vert}(P)$, and the parameters $k, m \in \mathbb{N}$ will be fixed later. It will be shown that, for a sufficiently large $k$ and $m$, we have $P=\left(p_{0}, p_{1}, p_{2}\right)_{\geq 0}$. Let us first show that there exists $\varepsilon \in\left(0, \varepsilon_{0}\right]$ such, for all sufficiently large $k \in \mathbb{N}$, we have

$$
U(P, \varepsilon, \rho) \cap\left(p_{1}, p_{2}\right)_{\geq 0} \subseteq P \cup\left(\bigcup_{v \in \operatorname{vert}(P)}\left(v-\operatorname{int} S_{\rho}(P, v)\right)\right)
$$

Let us consider an arbitrary $x \in U\left(P, \varepsilon_{0}, \rho\right)$.

Case 1: $x \in U_{P}\left(P, \varepsilon_{0}, \rho\right)=P$. Clearly, $x$ belongs to the left- and right-hand sides of (7.7).

Case 2: $x \in U_{F}\left(P, \varepsilon_{0}, \rho\right)$ for some $F \in \mathcal{F}_{2}(P)$. We have $p_{2}(x) \leq 0$ with equality if and only if $x \in F$. Consequently, $U_{F}\left(P, \varepsilon_{0}, \rho\right) \cap\left(p_{2}\right)_{\geq 0} \subseteq P$.

Case 3: $x \in U_{I}\left(P, \varepsilon_{0}, \rho\right)$ for some $I \in \mathcal{F}_{1}(P)$. If $x \in I$ and $v \in \operatorname{vert}(P) \backslash \operatorname{vert}(I)$, then at least one of the two facets $F$ from $\mathcal{F}_{2}(I, P)$ satisfies $v \notin F$, which yields $f_{v}(x)=0$. Consequently,

$$
\max _{v \in \operatorname{vert}(P) \backslash \operatorname{vert}(I)}\left|f_{v}(x)\right|=0 \quad \forall x \in I .
$$


We show that there exists $\beta_{I}>0$ is such that

$$
\beta_{I} \leq \max _{w \in \operatorname{vert}(I)}\left|f_{w}(x)\right| \quad \forall x \in U_{I}\left(P, \varepsilon_{0}, \rho\right) .
$$

Choose $x \in U_{I}\left(P, \varepsilon_{0}, \rho\right)$. If $x \in \operatorname{vert}(I)$, then $f_{w}(x)>0$ for $w=x$. If $x \in \operatorname{relint} I$, then $f_{w}(x)>0$ for every $w \in \operatorname{vert}(I)$. Now assume that $x \in U_{I}\left(P, \varepsilon_{0}, \rho\right) \backslash I$. We fix arbitrary $w \in \operatorname{vert}(I)$ and $F \in \mathcal{F}_{2}(P) \backslash \mathcal{F}_{2}(w, P)$. Consider the subcase $F \cap I=\emptyset$. By the definition of $U_{I}\left(P, \varepsilon_{0}, \rho\right)$, we have $x \in I+\mathbb{B}^{3}\left(o, \varepsilon_{0}\right)$, and by (7.2), we obtain $q_{F}(P, x) \neq 0$. Consider the subcase $F \cap I \neq \emptyset$. We denote by $v$ the endpoint of $I$ distinct from $w$. Then $F \in \mathcal{F}_{2}(v, P)$. Let us show that $q_{F}(P, x) \neq 0$ by contradiction. Assume the contrary, i.e., that $q_{F}(P, x)=0$. Then $F \in \mathcal{F}^{-}(P, x)$. Furthermore, $\mathcal{F}_{2}(I, P) \subseteq \mathcal{F}^{-}(P, x)$ by the definition of $U_{I}\left(P, \varepsilon_{0}, \rho\right)$. Taking into account the above relations together with $\mathcal{F}_{2}(I, P) \subseteq \mathcal{F}_{2}(v, P)$ and $F \in \mathcal{F}_{2}(v, P)$, we deduce $\mathcal{F}_{2}(I, P) \cup\{F\} \subseteq \mathcal{F}^{-}(P, x) \cap \mathcal{F}_{2}(v, P)$. Hence, $\left|\mathcal{F}^{-}(P, x) \cap \mathcal{F}_{2}(v, P)\right| \geq 3$, which implies that (5.3) cannot be fulfilled, a contradiction. Summarizing, we get

$$
\max _{w \in \operatorname{vert}(I)}\left|f_{w}(x)\right|>0 \quad \forall x \in U_{I}\left(P, \varepsilon_{0}, \rho\right),
$$

which yields the existence of $\beta_{I}$ satisfying (7.9). By Proposition 6.3(III),

$$
b_{w, l}(x)=0 \quad \forall w \in \operatorname{vert}(I) \forall x \in I .
$$

If $x \in \operatorname{aff} I \backslash I$, one has $x \notin S_{\rho}(P, w)$ for some vertex of $w \in \operatorname{vert}(I)$. Consequently, $U_{I}\left(P, \varepsilon_{0}, \rho\right) \cap$ aff $I=I$. By Proposition 6.3(III) we get

$$
b_{w, l}(x)<0 \quad \forall w \in \operatorname{vert}(I) \forall x \in U_{I}\left(P, \varepsilon_{0}, \rho\right) \backslash I .
$$

We also notice that $\max _{v \in \operatorname{vert}(P) \backslash \operatorname{vert}(I)}\left|f_{v}(x)\right|$ and $b_{w, l}(x)$ are semi-algebraic functions and $U_{I}\left(P, \varepsilon_{0}, \rho\right)$ is a semi-algebraic set. Hence, taking into account (7.8), (7.10), and (7.11) and applying Theorem 3.1, we obtain the existence of $k_{I} \in \mathbb{N}$ and $\gamma_{I}>0$ such that

$$
\max _{v \in \operatorname{vert}(P) \backslash \operatorname{vert}(I)} f_{v}(x)^{2 k_{I}} \leq \gamma_{I} \min _{w \in \operatorname{vert}(I)}\left|b_{w, l}(x)\right|
$$

for every $x \in U_{I}\left(P, \varepsilon_{0}, \rho\right)$. In view of (7.8), we can choose $\varepsilon_{I} \in\left(0, \varepsilon_{0}\right]$ such that, for every $x \in U_{I}\left(\varepsilon_{I}, \rho\right)$, one has

$$
\max _{v \in \operatorname{vert}(P) \backslash \operatorname{vert}(I)}\left|f_{v}(x)\right| \leq \frac{\beta_{I}}{2} .
$$

We also assume that $k$ is large enough so that the inequality

$$
\alpha_{l}|\operatorname{vert}(P)| \gamma_{I} 2^{-2 k}\left(\frac{2}{\beta_{I}}\right)^{2 k_{I}} \leq \frac{1}{2}
$$

is fulfilled. 
Then, for every $x \in U_{I}\left(P, \rho, \varepsilon_{I}\right)$ and every $k \in \mathbb{N}$ with $k \geq k_{I}$, we obtain

$$
\begin{aligned}
& \left|\sum_{v \in \operatorname{vert}(P) \backslash \operatorname{vert}(I)} f_{v}(x)^{2 k} b_{v, l}(x)\right| \\
& \stackrel{(7.3)}{\leq} \alpha_{l} \sum_{v \in \operatorname{vert}(P) \backslash \operatorname{vert}(I)} f_{v}(x)^{2 k} \\
& \leq \alpha_{l}|\operatorname{vert}(P)| \max _{v \in \operatorname{vert}(P) \backslash \operatorname{vert}(I)} f_{v}(x)^{2 k} \\
& \stackrel{(7.12)}{\leq} \alpha_{l}|\operatorname{vert}(P)| \gamma_{I} \min _{w \in \operatorname{vert}(I)}\left|b_{w, l}(x)\right| \max _{v \in \operatorname{vert}(P) \backslash \operatorname{vert}(I)} f_{v}(x)^{2\left(k-k_{I}\right)} \\
& \stackrel{(7.13)}{\leq} \alpha_{l}|\operatorname{vert}(P)| \gamma_{I}\left(\frac{\beta_{I}}{2}\right)^{2\left(k-k_{I}\right)} \min _{w \in \operatorname{vert}(I)}\left|b_{w, l}(x)\right| \\
& \stackrel{(7.9)}{\leq} \alpha_{l}|\operatorname{vert}(P)| \gamma_{I} 2^{-2 k}\left(\frac{2}{\beta_{I}}\right)^{2 k_{I}} \min _{w \in \operatorname{vert}(I)}\left|b_{w, l}(x)\right| \max _{w \in \operatorname{vert}(I)} f_{w}(x)^{2 k} \\
& \stackrel{\text { (7.14) }}{\leq} \frac{1}{2} \min _{w \in \operatorname{vert}(I)}\left|b_{w, l}(x)\right| \max _{w \in \operatorname{vert}(I)} f_{w}(x)^{2 k} \\
& \leq \frac{1}{2} \min _{w \in \operatorname{vert}(I)}\left|b_{w, l}(x)\right| \sum_{w \in \operatorname{vert}(I)} f_{w}(x)^{2 k} \\
& \leq \frac{1}{2} \sum_{w \in \operatorname{vert}(I)} f_{w}(x)^{2 k}\left|b_{w, l}(x)\right| \text {. }
\end{aligned}
$$

Consequently, for $x$ and $k$ as above, we get

$$
\begin{aligned}
p_{1}(x) & \leq \sum_{w \in \operatorname{vert}(I)} f_{w}(x)^{2 k} b_{w, l}(x)+\left|\sum_{v \in \operatorname{vert}(P) \backslash \operatorname{vert}(I)} f_{v}(x)^{2 k} b_{v, l}(x)\right| \\
& \leq \sum_{w \in \operatorname{vert}(I)} f_{w}(x)^{2 k} b_{w, l}(x)+\frac{1}{2} \sum_{w \in \operatorname{vert}(I)} f_{w}(x)^{2 k}\left|b_{w, l}(x)\right| \\
& \stackrel{\text { (7.11) }}{=} \frac{1}{2} \sum_{w \in \operatorname{vert}(I)} f_{w}(x)^{2 k} b_{w, l}(x) \stackrel{(7.11)}{\leq} 0
\end{aligned}
$$

with equality $p_{1}(x)=0$ if and only if $x \in I$.

Case 4: $x \in U_{v}^{\prime}\left(P, \varepsilon_{0}, \rho\right)$ for some $v \in \operatorname{vert}(P)$. By Proposition 6.3(V) and the definition of $U_{v}^{\prime}\left(P, \varepsilon_{0}, \rho\right)$ we have

$$
b_{v, l}(x)<0 \quad \forall x \in U_{v}^{\prime}\left(P, \varepsilon_{0}, \rho\right) \backslash\{v\} .
$$

In view of (7.2), the definition of $f_{v}(x)$, and the inclusion $U_{v}^{\prime}\left(P, \varepsilon_{0}, \rho\right) \subseteq \mathbb{B}^{3}\left(v, \varepsilon_{0}\right)$, there exists $\beta_{v}>0$ such that

$$
f_{v}(x) \geq \beta_{v} \quad \forall x \in U_{v}^{\prime}\left(P, \varepsilon_{0}, \rho\right) .
$$


On the other hand,

$$
\max _{w \in \operatorname{vert}(P) \backslash\{v\}}\left|f_{w}(v)\right|=0 .
$$

Notice that $\max _{w \in \operatorname{vert}(P) \backslash\{v\}}\left|f_{w}(x)\right|$ and $b_{v, l}(x)$ are semi-algebraic function and that $U_{v}^{\prime}\left(P, \varepsilon_{0}, \rho\right)$ is a semi-algebraic set. Thus, taking into account (7.15) and (7.17) and applying Theorem 3.1, we find $\gamma_{v}>0$ and $k_{v} \in \mathbb{N}$ such that

$$
\max _{w \in \operatorname{vert}(P) \backslash\{v\}}\left|f_{w}(x)\right|^{2 k_{v}} \leq \gamma_{v}\left|b_{v, l}(x)\right|
$$

for every $x \in U_{v}^{\prime}\left(P, \varepsilon_{0}, \rho\right)$. In view of (7.17), we can choose $\varepsilon_{v} \in\left(0, \varepsilon_{0}\right]$ such that

$$
\max _{w \in \operatorname{vert}(P) \backslash\{v\}}\left|f_{w}(x)\right| \leq \frac{\beta_{v}}{2}
$$

for every $x \in U_{v}^{\prime}\left(P, \varepsilon_{v}, \rho\right)$. We assume that $k$ is large enough so that

$$
\alpha_{l}|\operatorname{vert}(P)| \gamma_{v}\left(\frac{\beta_{v}}{2}\right)^{-2 k_{v}} 2^{-2 k} \leq \frac{1}{2}
$$

Then, for every $x \in U_{v}^{\prime}\left(P, \varepsilon_{v}, \rho\right)$ and $k \geq k_{v}$, as above, we obtain

$$
\begin{aligned}
& \left|\sum_{w \in \operatorname{vert}(P) \backslash\{v\}} f_{w}(x)^{2 k} b_{w, l}(x)\right| \\
& \quad \stackrel{(7.3)}{\leq} \alpha_{l}|\operatorname{vert}(P)| \max _{w \in \operatorname{vert}(P) \backslash\{v\}}\left|f_{w}(x)\right|^{2 k} \\
& \quad \stackrel{(7.18)}{\leq} \alpha_{l}|\operatorname{vert}(P)| \gamma_{v}\left|b_{v, l}(x)\right| \max _{w \in \operatorname{vert}(P) \backslash\{v\}}\left|f_{w}(x)\right|^{2\left(k-k_{v}\right)} \\
& \quad \stackrel{(7.19)}{\leq} \alpha_{l}|\operatorname{vert}(P)| \gamma_{v}\left(\frac{\beta_{v}}{2}\right)^{2\left(k-k_{v}\right)}\left|b_{v, l}(x)\right| \\
& \quad \stackrel{(7.16)}{\leq} \alpha_{l}|\operatorname{vert}(P)| \gamma_{v}\left(\frac{\beta_{v}}{2}\right)^{2\left(k-k_{v}\right)}\left(\beta_{v}\right)^{-2 k} f_{v}(x)^{2 k}\left|b_{v, l}(x)\right| \\
& \quad=\alpha_{l}|\operatorname{vert}(P)| \gamma_{v}\left(\frac{\beta_{v}}{2}\right)^{-2 k_{v}} 2^{-2 k} f_{v}(x)^{2 k}\left|b_{v, l}(x)\right| \\
& \quad \stackrel{(7.20)}{\leq} \frac{1}{2} f_{v}(x)^{2 k}\left|b_{v, l}(x)\right| .
\end{aligned}
$$

Hence, taking into account (7.15) and (7.16), we obtain that, for $k$ satisfying (7.20) and all $x \in U_{v}^{\prime}\left(P, \varepsilon_{v}, \rho\right)$, we have $p_{1}(x) \leq 0$ with equality if and only if $x=v$. 
Consequently, for $x$ and $k \geq k_{v}$, as above, we obtain

$$
\begin{aligned}
p_{1}(x) & \leq f_{v}(x)^{2 k} b_{v, l}(x)+\left|\sum_{w \in \operatorname{vert}(P) \backslash\{v\}} f_{w}(x)^{2 k} b_{w, l}(x)\right| \\
& \leq f_{v}(x)^{2 k} b_{v, l}(x)+\frac{1}{2} f_{v}(x)^{2 k}\left|b_{v, l}(x)\right| \\
\stackrel{(7.15)}{=} & \frac{1}{2} f_{v}(x)^{2 k} b_{v, l}(x) \stackrel{(7.15)}{\leq} 0
\end{aligned}
$$

with equality $p_{1}(x)=0$ if and only if $x=v$.

Case 5: $x \in U_{v}\left(P, \varepsilon_{0}, \rho\right) \backslash U_{v}^{\prime}\left(P, \varepsilon_{0}, \rho\right)$ for some $v \in \operatorname{vert}(P)$. By the definition of $U_{v}\left(P, \varepsilon_{0}, \rho\right)$ and $U_{v}^{\prime}\left(P, \varepsilon_{0}, \rho\right)$, we easily see that $x \in v-\operatorname{int} S_{\rho}(P, v)$. Thus, $U_{v}\left(P, \varepsilon_{0}, \rho\right) \backslash U_{v}^{\prime}\left(P, \varepsilon_{0}, \rho\right) \subseteq v-$ int $S_{\rho}(P, v)$.

By means of the arguments given in the above five cases we verified that (7.7) holds if $k \geq k_{v}, k \geq k_{I}, \varepsilon \leq \varepsilon_{v}, \varepsilon \leq \varepsilon_{I}$ for all $v \in \operatorname{vert}(P)$ and $I \in \mathcal{F}_{1}(P)$ and that inequalities (7.14) and (7.20) are fulfilled. By Proposition 5.1, there exists $\delta>0$ such that $P+\mathbb{B}^{3}(o, \delta) \subseteq U(P, \varepsilon, \rho)$. By condition $\mathcal{A}(P)$, we can choose $m \in \mathbb{N}$ such that $\left(p_{0}\right)_{\geq 0} \subseteq P+\mathbb{B}^{3}(o, \delta)$. Thus, for $m$, as above, we obtain

$$
\begin{aligned}
\left(p_{0}, p_{1}, p_{2}\right)_{\geq 0} & =\left(p_{0}, p_{1}, p_{2}\right)_{\geq 0} \cap\left(P+\mathbb{B}^{3}(o, \delta)\right) \subseteq\left(p_{0}, p_{1}, p_{2}\right)_{\geq 0} \cap U(P, \varepsilon, \rho) \\
& \stackrel{(7.7)}{\subseteq}\left(p_{0}\right)_{\geq 0} \cap\left(P \cup \bigcup_{v \in \operatorname{vert}(P)}\left(v-\operatorname{int} S_{\rho}(P, v)\right)\right) \\
& =\left(\left(p_{0}\right)_{\geq 0} \cap P\right) \cup\left(\left(p_{0}\right)_{\geq 0} \cap \bigcup_{v \in \operatorname{vert}(P)}\left(v-\operatorname{int} S_{\rho}(P, v)\right)\right) \\
& \stackrel{(6.2)}{=} P \cup \operatorname{vert}(P)=P .
\end{aligned}
$$

Thus, $\left(p_{0}, p_{1}, p_{2}\right)_{\geq 0}=P$.

7.2 The Case of Unbounded Polyhedra

The following is a modified version of Łojasiewicz's Inequality.

Lemma 7.2 Let $A$ be a bounded and closed semi-algebraic set in $\mathbb{R}^{d}$. Let $f(x)$ and $g(x)$ be continuous semi-algebraic functions on A satisfying

$$
\{x \in A: f(x)=0\} \subseteq\{x \in A: g(x)=0\} .
$$

Then there exists $n^{\prime} \in \mathbb{N}$ such that, for every $n \in \mathbb{N}$ with $n \geq n^{\prime}$, there is a constant $\lambda>0$ satisfying $|g(x)|^{n} \leq \lambda|f(x)|$ for every $x \in A$.

Proof By Theorem 3.1 we can fix $n^{\prime} \in \mathbb{N}$ and $\lambda^{\prime}>0$ such that $|g(x)|^{n^{\prime}} \leq \lambda^{\prime}|f(x)|$ for every $x \in A$. Let $\mu \geq 0$ be an upper bound of $|g(x)|$ on $A$. Then, for every $n \in \mathbb{N}$ with $n \geq n^{\prime}$, we have $|g(x)|^{n} \leq \mu^{n-n^{\prime}} \lambda^{\prime}|f(x)|$, so that we may set $\lambda:=\mu^{n-n^{\prime}} \lambda^{\prime}$. 
Proposition 7.3 Let $d \in \mathbb{N}$ and $d \geq 2$. Assume that, for every $n=1, \ldots, d$ and every $n$-polytope $P$ in $\mathbb{R}^{d}$, there exist $p_{0}(x), \ldots, p_{n-1}(x) \in \mathbb{R}[x]$ satisfying

$$
P=\left\{x \in \text { aff } P: p_{0}(x) \geq 0, \ldots, p_{n-1}(x) \geq 0\right\}
$$

and $p_{0}(x)>0$ for all $x \in P \backslash \operatorname{vert}(P)$. Then, for every $d$-dimensional polyhedron $P$ in $\mathbb{R}^{d}$, there exist $f_{0}(x), \ldots, f_{d-1}(x) \in \mathbb{R}[x]$ satisfying $P=\left(f_{0}, \ldots, f_{d-1}\right)_{\geq 0}$.

Proof Consider an arbitrary unbounded $d$-polyhedron $P$ in $\mathbb{R}^{d}$. Using the same arguments as in the beginning of the proof of Theorem 1.1, we may restrict ourselves to the case where $P$ is line-free. The set hom $(P)$ is a $(d+1)$-dimensional pointed polyhedral cone. Since hom $(P)$ is pointed, one can determine a hyperplane $H^{\prime}$ in $\mathbb{R}^{n+1}$ such that $P^{\prime}:=\operatorname{hom}(P) \cap H^{\prime}$ is bounded and $\operatorname{hom}(P)=\operatorname{cone}\left(P^{\prime}\right)$. Let $u^{\prime} \in \mathbb{R}^{d+1} \backslash\{o\}$ be the normal of $H^{\prime}$ with $H^{\prime}=\left\{x \in \mathbb{R}^{d+1}:\left\langle x, u^{\prime}\right\rangle=1\right\}$. Let us consider polynomials $p_{0}(x), \ldots, p_{d-1}(x) \in \mathbb{R}[x]$ such that $P=\left\{x \in\right.$ aff $P^{\prime}: p_{0}(x) \geq$ $\left.0, \ldots, p_{d-1}(x) \geq 0\right\}$ and $p_{0}(x)>0$ for every $x \in P \backslash \operatorname{vert}(P)$. Without loss of generality we may assume that $p_{0}(x), \ldots, p_{d-1}(x)$ are homogeneous. We define

$$
f(x):=\prod_{v \in \operatorname{vert}\left(P^{\prime}\right)}\left(\|x\|^{2}\|v\|^{2}-\langle x, v\rangle^{2}\right) .
$$

By Lemma 7.2 applied to $f(x)$ and $p_{0}(x)$ restricted to $P^{\prime}$, there exist $\lambda>0$ and $l \in \mathbb{N}$ satisfying $l \operatorname{deg} f(x)>\operatorname{deg} p_{0}(x)$ and $\lambda p_{0}(x)-f(x)^{l} \geq 0$ for every $x \in P^{\prime}$ (where deg stands for degree). Define $f_{0}(x):=\lambda p_{0}(x)\left\langle x, u^{\prime}\right\rangle^{k_{0}}-f(x)^{l}$, where $k_{0} \in \mathbb{N}$ is chosen in such a way that $f_{0}$ is homogeneous, i.e., $k_{0}$ is determined from the equality

$$
k_{0}+\operatorname{deg} p_{0}(x)=l \operatorname{deg} f(x) .
$$

We also set $f_{i}(x):=p_{i}(x)\left\langle x, u^{\prime}\right\rangle^{k_{i}}$ for $i=1, \ldots, d-1$, where $k_{1}, \ldots, k_{d-1} \in\{1,2\}$ are chosen in such a way that $f_{1}(x), \ldots, f_{d-1}(x)$ have even degrees. By construction, the polynomials $f_{0}(x), \ldots, f_{d-1}(x)$ are homogeneous, have even degrees, and satisfy

$$
\operatorname{hom}(P) \cup(-\operatorname{hom}(P))=\left\{x \in \mathbb{R}^{d+1}: f_{0}(x) \geq 0, \ldots, f_{d-1}(x) \geq 0\right\} .
$$

Hence, $\left\{x \in \operatorname{aff} P: f_{0}(x) \geq 0, \ldots, f_{d-1}(x) \geq 0\right\}=(\operatorname{hom}(P) \cup(-\operatorname{hom}(P))) \cap$ $($ aff $P)=P$.

Theorem 1.1 is a direct consequence of Theorem 7.1 and Proposition 7.3.

Acknowledgements We would like to thank the referee for helpful suggestions which improved the presentation of the paper.

\section{References}

1. Andradas, C., Bröcker, L., Ruiz, J.M.: Constructible Sets in Real Geometry. Ergebnisse der Mathematik und ihrer Grenzgebiete (3) [Results in Mathematics and Related Areas (3)], vol. 33. Springer, Berlin (1996) 
2. Averkov, G., Henk, M.: Representing simple $d$-dimensional polytopes by $d$ polynomial inequalities. Math. Program., Ser. A (2009). doi:10.1007/s10107-009-0280-y

3. Averkov, G.: Representing elementary semi-algebraic sets by a few polynomial inequalities: a constructive approach. Submitted (2008)

4. Bochnak, J., Coste, M., Roy, M.-F.: Real Algebraic Geometry. Ergebnisse der Mathematik und ihrer Grenzgebiete (3) [Results in Mathematics and Related Areas (3)], vol. 36. Springer, Berlin (1998). Translated from the 1987 French original, Revised by the authors

5. Bernig, A.: Constructions for the theorem of Bröcker and Scheiderer, Master's Thesis, Universität Dortmund (1998)

6. Bosse, H., Grötschel, M., Henk, M.: Polynomial inequalities representing polyhedra. Math. Program. 103(1A), 35-44 (2005)

7. Bröcker, L.: Remarks on the description of polytopes by few polynomials. Manuscript (2008)

8. Gruber, P.M.: Convex and Discrete Geometry. Grundlehren der Mathematischen Wissenschaften [Fundamental Principles of Mathematical Sciences], vol. 336. Springer, Berlin (2007)

9. Henk, M.: Polynomdarstellungen von Polyedern. Jahresber. Dtsch. Math.-Ver. 109(2), 51-69 (2007)

10. Helton, W.J., Nie, J.: Structured semidefinite representation of some convex sets. Preprint: arXiv:0802.1766v1 (2008)

11. Laurent, M.: Sums of squares, moment matrices and optimization over polynomials. In: Putinar, M., Sullivant, S. (eds.) Emerging Applications of Algebraic Geometry (2008)

12. Łojasiewicz, S.: Sur le problème de la division. Stud. Math. 18, 87-136 (1959)

13. Prestel, A.: Model theory for the real algebraic geometer. Dottorato di ricerca in matematica, Istituti editoriali e poligrafici internazionali, Dipartimento di matematica dell'universitá di Pisa (1998)

14. Schneider, R.: Convex Bodies: The Brunn-Minkowski Theory. Encyclopedia of Mathematics and Its Applications, vol. 44. Cambridge University Press, Cambridge (1993)

15. Ziegler, G.M.: Lectures on Polytopes. Graduate Texts in Mathematics, vol. 152. Springer, New York (1995) 